\title{
Individual and community psychological experiences of the COVID-19 pandemic: The state of emergency in Portugal
}

\author{
Alice Murteira Morgado ${ }^{1,2}$ (D) Joana $\mathrm{Cruz}^{2} \cdot$ Maria Manuela Peixoto ${ }^{2}$ \\ Accepted: 24 March 2021 / Published online: 1 April 2021 \\ (C) The Author(s), under exclusive licence to Springer Science+Business Media, LLC, part of Springer Nature 2021
}

\begin{abstract}
Individuals have different ways of coping with crisis. Individual factors, family and contextual features, and community support may influence how individuals feel, think and act during a crisis. COVID-19 was an unexpected pandemic that forced many European countries to take confinement measures and restrict social face to face interactions. This study is an effort to understand how Portuguese residents dealt with the pandemic during the first confinement period, considering different sociodemographic characteristics and trauma exposure perceptions. Five hundred and five adults, between 18 and 79 years old participated in this study via an online self-report assessment protocol. Sociodemographic characteristics such as gender, age, marital status, employment status, and caring responsibilities had an impact on individuals' perceptions regarding their exposure to the pandemic and their quality of life. Perceived exposure to the pandemic was found to predict quality of life in the physical, psychological, and environmental domains. Results have practical implications for European and local policy-making, as well as for targeting psychological interventions for those whose mental health has been negatively affected by the pandemic and for those who may become more affected if confinement measures are implemented again.
\end{abstract}

Keywords COVID-19 · Mental health · Trauma exposure · Quality of life

The COVID-19 pandemic presents significant challenges to individual and collective functioning, calling for an extensive analysis of its consequences as a crisis-triggering event. Indeed, it is paramount to understand what can be done to face the specific risk factors of the pandemic (Duan \& Zhu, 2020) and conceptualising the COVID-19 pandemic as a disaster may reveal particularly useful (Morgado, 2020). This study offers an outlook on the psychosocial consequences of the pandemic for the Portuguese population, where the COVID19 pandemic involved an initial generalised lockdown that lasted 2 months for a significant part of the population.

As suggested by Norris and Wind (2010), the assessment of a disaster should take into account the magnitude of its consequences, especially if there is loss of life (and, consequently, traumatic bereavement), threats to life, witnessing of

Alice Murteira Morgado

alice.morgado@outlook.pt

1 Escola Secundária José Falcão, Avenida Afonso Henriques, 3000-011 Coimbra, Portugal

2 Psychology for Positive Development Research Center (CIPD), Universidade Lusíada (Porto), Lisbon, Portugal horror, and loss of resources. Morgado (2020) further suggests that psychological research should focus on preventing disasters as experiences that are out of individual and community control and that should be addressed both individually and collectively.

Having been exposed to a disaster does not necessarily lead to psychopathological symptoms, but it may have a profound impact on individuals' belief systems with regard to personal resources and trust in social systems (Pérez-Sales et al., 2005). When these individual core beliefs, which allow to give meaning to the world, are violated, individuals are at higher risk to develop severe psychopathological symptoms (Milman et al., 2020a) and experience higher coronavirus anxiety (Milman et al., 2020b). As such, the concept of disaster may easily apply to the COVID-19 pandemic, given its local and global implications, and the necessary efforts to mitigate any psychological, social, and economic consequences. Indeed, the pandemic is being experienced by a significant number of individuals and communities, involving, for an indefinite period of time, serious challenges, not just for individuals, but also for families, communities, societies, governments and economies (Benight et al., 2010; Duan \& Zhu, 2020; Hoffman \& Kruczek, 2011; Raphael \& Maguire, 2010; Venuleo et al., 2020). Moreover, psychosocial interventions targeting health 
promotion may need to focus on how individuals reconstruct their meaning of the world, in order to restore individual core beliefs, through processes of sense of coherence, predictability, metaphors, and narratives (Castiglioni \& Gaj, 2020).

Experiences of psychological crisis may include emotional, behavioural, and physical symptoms of stress. The timeframe of the COVID-19 pandemic may involve the continuation of these stress reactions in a relatively long period of time, that may lead to psychopathological disorders such as Posttraumatic Stress Disorder (PTSD), Anxiety Disorders, and Depression (Brooks et al., 2020; Duan \& Zhu, 2020; Rajukmar, 2020). These may represent an added strain and burden, not just for individuals, but for mental health services and professionals during and after the pandemic.

In the context of the COVID-19 pandemic, a series of specific stressors need to be considered, as they may contribute to increased risk for traumatisation: (1) the contagiousness and uncertainty around transmission routes, treatment, vaccination, and mortality rates; (2) psychological challenges of confinement and social isolation measures; (3) sudden or unexpected social, financial and occupational shifts or difficulties; and (4) attitudes towards and concerns with vulnerable populations, such as the elderly, people with chronic diseases, people with history of mental health problems, pregnant women, and people exposed to increased risk of infection, such as health professionals, frontline workers, etc. (Biviá-Roig et al., 2020; Duan \& Zhu, 2020; Hou et al., 2020; Pariente et al., 2020; Rajukmar, 2020; Zhang et al., 2020). Known COVID-19 characteristics may also lead to symptoms that are typically attributed to Obsessive-Compulsive Disorder or Anxiety Disorders such as Agoraphobia and Social Anxiety, for example (Coelho et al., 2020). Indeed, despite being strongly encouraged by authorities as protective measures against COVID-19 and considered adaptive in more critical stages of the pandemic, constant cleaning and disinfection behaviours, as well as social avoidance and withdrawal from activities may become pathological once the disease no longer represents a public health threat (Morgado, 2020). In this context, Asmundson and Taylor (2020) also highlight the risk of increasing health anxiety symptoms, that may result either in excessive demand and hoarding of health services or in its avoidance when they are manifestly necessary. Another specific mental health challenge regards the risk of traumatic grief and bereavement, not just due to the known effects of multiple losses for individuals, families, and communities (Verdery \& Smith-Greenaway, 2020), but also considering the existing limitations to death-related rituals, such as funerals and other solemn ceremonies that typically allow for the ventilation of intense grief emotions. The existing restraints to these rituals may cause significant stress on individuals, that may be limited in their expression of grief and in the attribution of meaning to the loss, both of which are critical for normative grief responses (Neimeyer et al., 2010).
Studies on resilience following traumatic experiences (e.g., Cox \& Perry, 2011; Schulenberg, 2016) suggest that positive post-crisis outcomes involve positive psychological functioning and well-being, more than just the absence of psychopathology. Resilience is an important factor for the continuation of a positive life (Bonanno \& Gupta, 2010; Cox \& Perry, 2011; Raphael \& Maguire, 2010) and even for posttraumatic growth (Hoffman \& Kruczek, 2011; Schulenberg, 2016). Community resilience has been associated withpositive trajectories as it results in better coordination and organisation of resources to support affected networks and to empower individuals and communities (Gil-Rivas \& Kilmer, 2016; Norris et al., 2008). The active role of individuals and communities allows for the involvement and participation on individual and collective meaning-making processes about the traumatic events (Norris \& Wind, 2010; Schulenberg, 2016). Social and emotional support have also been found to protect individuals from psychological suffering and distress and to promote well-being positive mental health outcomes in the context of the COVID-19 pandemic (Pedrosa et al., 2020; Zysberg \& Zisberg, 2020). Thus, both individual and community resources should enhance the acknowledgement of stress reactions as acceptable and valid reactions during distressful experiences such as the COVID-19 pandemic, promoting a sense of coherence that allows to restore meaning and the individual's core beliefs system (Castiglioni \& Gaj, 2020).

Considering the above, it becomes especially timely to consider both community consequences and increased individual stress arising from the COVID-19 pandemic. Morgado (2020) suggests that such consequences may be either aggravated or moderated by the degree of coordination of governmental organizations, mental health services, first aid response systems, and also the portrayal of the situation by the media. Indeed, appropriate coordination has the potential to reassure individuals and communities, whereas disorganised, ineffective or non-existing responses may lead to a sense of helplessness, lack of support, and community disruption that may increase the risk for individual stress/psychopathology and may interfere negatively with adaptive functioning (Morgado, 2020).

\section{The Case of Portugal}

The effectiveness of coordinated responses provided by healthcare services, but also by communities, governments, social support agencies, and media outlets is crucial to address the individual and collective mental health risks that are in place due to the COVID-19 pandemic worldwide. Throughout Europe, different countries have adopted different responses to this crisis, based on their own infectiousness rates, cultural backgrounds and priorities. Approaches ranged from recommendations on social distancing, use of face masks 
and safety rules for circulation in public spaces, to full country lockdowns (European Centre for Disease Prevention and Control, 2020). Specifically, in Portugal, the first COVID-19 case was diagnosed on 2nd March, 2020, with the government implementing a state of emergency soon after, on 16th March, 2020. This measure meant that schools were closed, most workers had to stay home, most public and private services and businesses were closed to the public, and the overall recommendation was to stay home and avoid any unnecessary travelling or face-to-face contacts with others. The state of emergency was renewed for six more weeks, having been progressively shifted into state of calamity on 2nd May, 2020. At this point, some services and businesses were allowed to reopen with several rules and restrictions, but most workers and families were still spending most of their time at home. Generally, the country started returning to "the new normality" in June, with some regions being more restricted than others due to the number of new cases (for example, the capital city, Lisboa).

As important as these measures were to stop the uncontrolled community transmission, they had a significant impact on individual, family, and community functioning. Children and adolescents continued the ongoing school level via online synchronous classes with their teachers with the support of television broadcasted lectures promoted by the government. Investment was made, nationally and locally, to ensure that every pupil, from elementary to high school, would have a technologic tool and internet connection to participate in synchronous classes with their teachers. This measure was no able to reach all pupils and was delivered with delay for some others.

The active adult population was either working remotely from home (if their role was compatible with remote work), put in lay-off, working extra time with different shifts (especially if they were considered "key-workers" for health, safety, and other essential activities) or on family leave to support caregivers with special needs or below 12 years of age.

The elderly, prisoners and adults in care became especially isolated, with care homes, prisons and health institutions forbidding external visitors for health and safety reasons. The same reasons made it impossible for individuals to pay visits to sick relatives or to say goodbye to loved ones if they were critically ill.

Those who stayed home were deprived from social support systems that were suspended, like hygiene, cleaning and feeding services at home. Overall, most tertiary activities were closed or highly restricted in their functioning, which meant a considerable financial and occupational loss for a big part of the Portuguese population.

On the other hand, public health services were working around the clock. Health care services were restructured to respond to testing and treating COVID-19 patients. To ensure that all residents had access to information and help in this matter, the government created a phone-line to respond to: (a) questions about COVID-19, including prevention and testing procedures; (b) needs regarding COVID-19 related symptoms to be addressed by a health professional; (c) needs regarding non-COVID-19 matters to be addressed by a health professional; (d) psychological support. There were also daily press conferences to share data from the pandemic and to inform citizens about measures being taken to support communities and individuals.

The challenges of the pandemic were especially demanding for 2 months in Portugal, after which, despite being eased, were not eliminated and will not be for an indefinite period of time, until the COVID-19 pandemic is fully managed and resolved. There are no known studies that have assessed the effects of this generalised experience of confinement with this magnitude, duration, and implications (Brooks et al., 2020). As such, the uncertainty, the passing of time, the isolation for some, and the extra work and burden of care for others have meant major unexpected shifts. The authors anticipate that these may have important psychological, physical, social, and financial implications.

\section{Objectives}

This paper presents a study intended to understand the impact of the state of emergency due to the pandemic on a sample of Portuguese residents, also looking at the influence of different sociodemographic characteristics on the participants' quality of life and trauma exposure perceptions. The aim is to identify the implications of such an unprecedented experience for the quality of life of different sociodemographic groups, to inform and design strategies that can promote the adaptive functioning and well-being of individuals and communities.

These objectives guided two major research questions. The first research question related to whether there are differences in quality of life and trauma exposure perceptions by the end of the first COVID-19 confinement period according to different sociodemographic characteristics: gender, age, marital status, employment status after the state of emergency, caregivers of those below and above 12 years of age, those potentially exposed to risk of infection at work and those who were not exposed to that occupational risk. We expect that, due to Portuguese cultural characteristics and political and health measures taken during the first confinement, women, the elderly, single people, the unemployed, those with caregiving responsibilities (especially of those below 12 years of age) and those potentially exposed to risk of infection at work, present lower scores in quality of life because of the higher risk of isolation, the burdens and responsibilities, as well as the lack of support (Pedrosa et al., 2020; Torres et al., 2018; Voicu et al., 2009).

The second research question addresses whether individual perceptions on trauma exposure predict quality of life in all its domains (physical, psychological, social, and environmental). We expect that that the perceived exposure to COVID-19 and 
the perceived support at individual and community levels contributes to explaining quality of life scores (Hoffman \& Kruczek, 2011; Morgado, 2020).

\section{Method}

\section{Participants}

The sample was composed of 505 adults (102 men and 401 women) between 18 and 79 years old (mean age $=42$ ) who lived in Portugal during the emergency state that was declared due to the COVID-19 pandemic. Participation in the study was voluntary and responses to the questionnaire were online, confidential, and anonymous. As such, this was a convenience sample, composed of all those who voluntarily filled the online form presented. The main sociodemographic characteristics as reported by participants in the sample are presented in Table 1. Participants reportedly lived in 20 different Portuguese districts, with the majority living in the two more densely populated districts: Lisboa (20\%) and Porto (25,3\%), Coimbra (17\%), Braga (6\%), and Setúbal $(5 \%)$ were the other three districts with more participants.

\section{Measures}

The assessment measures were all self-report questionnaires, focusing on the assessment of sociodemographic characteristics, quality of life, and trauma exposure perceptions. As such, the study protocol included three instruments: a sociodemographic questionnaire, the Trauma Exposure Checklist (Morgado et al., 2021), and the Portuguese version of the WHOQOL-BREF (Vaz Serra et al., 2006).

The sociodemographic questionnaire included questions about the individuals' characteristics (i.e. gender, age, education, work, family structure, etc.), as well as questions about any changes to their occupation or living arrangements due to the COVID-19 pandemic.

The Trauma Exposure Checklist (TEC), developed by Morgado et al. (2021) in the context of the COVID-19 pandemic comprises two self-report scales with a total of 24 items that may be answered on a 6-point Likert scale (1-completely disagree to 6-completely agree). The first scale, "self-perception of the exposure" includes 15 items that assess the factors "loss" and "coping" relating to the crisis-triggering event. The second scale considers "perceived community support" with 9 items grouped in "global measures" and "local measures". All scales presented good psychometric properties, with Cronbach alpha values ranging from .72 to 90 (Morgado et al., 2021).

The Portuguese version of the WHOQOL-BREF (Vaz Serra et al., 2006) is the adaptation of the World Health Organization Quality of Life Instrument, composed of 26
Table 1 Sociodemographic characteristics

Frequency

\begin{tabular}{|c|c|}
\hline \multicolumn{2}{|l|}{ Gender } \\
\hline Male & 102 \\
\hline Female & 401 \\
\hline \multicolumn{2}{|l|}{ Age } \\
\hline $18-24$ & 74 \\
\hline $25-34$ & 89 \\
\hline $35-44$ & 118 \\
\hline $45-54$ & 99 \\
\hline $55-64$ & 93 \\
\hline $65+$ & 26 \\
\hline \multicolumn{2}{|l|}{ Highest school level completed } \\
\hline Primary education (4 years) & 2 \\
\hline Basic education (9 years) & 16 \\
\hline Secondary education (12 years) & 98 \\
\hline University - Bachelor & 244 \\
\hline University - Master & 118 \\
\hline University - Doctorate & 22 \\
\hline \multicolumn{2}{|l|}{ Marital Status } \\
\hline Single & 181 \\
\hline Married/Civil partnership & 259 \\
\hline Divorced/Separated & 57 \\
\hline Widowed & 7 \\
\hline \multicolumn{2}{|l|}{ Dependents } \\
\hline No & 298 \\
\hline Yes & 207 \\
\hline At least one younger than 12 & 98 \\
\hline All above 12 years of age & 108 \\
\hline \multicolumn{2}{|l|}{ Exposure to risk of infection at work } \\
\hline No & 430 \\
\hline Yes & 71 \\
\hline Health professionals & 34 \\
\hline
\end{tabular}

items that assess one general factor on Health and Quality of Life along with 4 sub-domains: physical, psychological, social relations, and environment. The Portuguese version presented good psychometric properties, with Cronbach alpha values ranging from .64 to .87 for the domains, and .92 for the overall scale (Vaz Serra et al., 2006).

\section{Procedure}

Data was collected via web-survey. The Checklist for Reporting Results of Internet E-Surveys (Eysenbach, 2004) was used as a guide to prepare the online questionnaire (see appendix).

The only preconditions for participation were being an adult (aged 18+) and living in Portugal. The online questionnaire was announced through social media networks, mailing 
lists and websites to address a diverse range of public. Information about the aims of data collection was briefly described. Upon opening the link for the questionnaire participants were provided with a full description of the objectives, institutional framework, length and confidentiality issues. If adults chose to fill the questionnaire, they filled an online consent form and could start answering it. It was stated that participation was voluntary, with no incentives for participation being offered. No information that would allow to identify each participant individually was requested, hence granting the anonymity of all data. Participants were assured about the confidentiality and informed that their participation was voluntary. Items completion took between 10 and $12 \mathrm{~min}$. Scale administration occurred between the 7 th and the $23 \mathrm{rd}$ of May, 2020, a period when the Portuguese population was mostly confined due to the COVID-19 pandemic, with participants being instructed to answer based on their current experiences.

\section{Data Analysis}

Data was analysed with IBM SPSS v26, multivariate analysis of variance (MANOVA) and multiple regressions were used.

A series of multivariate analysis of variance (MANOVA) were conducted with seven sociodemographic characteristics as independent variables: gender, age group, marital status, employment status, caregivers, age group of people under care, and risk of infection at work. The WHOQOL-BREF dimensions (physical, psychological, social relations, environment) and TEC scales (loss, coping, local measures, and global measures) as dependent variables.

Normality of distribution, equality of variance, and univariate outliers were initially checked, with most variables meeting assumptions for analysis of variance, with only some small deviations from normality (Field, 2018). Absence of multivariate outliers was confirmed through the assessment of Mahalanobis Distances among participants, with no scores found above the critical chi-square value for each analysis $\left(\chi^{2}=18.47, \mathrm{df}=4, p=.001\right)$. Linearity was met for each group of the MANOVA, confirmed through the analysis of a scatterplot matrix between the dependent variables. Multicollinearity was checked through correlations among the dependent variables. The highest correlation between dependent variables was found between the physical and psychological domains of WHOQOL-BREF at .64, confirming the absence of multicollinearity (Field, 2018).

Equality of covariance matrices was checked through Box's M significance levels. In most tests, significance was above .05 , allowing to confirm homogeneity of covariance. Where significance was below .05, group sizes included more than 30 participants, each, making the MANOVA robust against violations of this assumption (Allen \& Bennett, 2008).
Four regression models, with TEC scale scores as predictors and WHOQOL-BREF dimensions as outcome variables were performed to test $\mathrm{H}_{8}$. A stepwise approach was chosen due to the exploratory nature of the study (Field, 2018).

Assumptions for these analyses were all met. An analysis of standard residuals was carried out on the data to identify any outliers, which indicated that participants 339, 411, and 493 needed to be removed. After removal of these outliers, analyses of standard residuals confirmed that the data contained no outliers (Std. Residual Min $=-3.26$, Std. Residual Max =2.49). Tests to see if the data met the assumption of collinearity indicated that multicollinearity was not a concern in any of the models tested (Tolerance values from 1 to .80 ; VIF ranged from 1 to 1.26 ). The data also met the assumption of independent errors (Durbin-Watson values $=$ $2.02,2.06,2.00,2.15)$. The histograms of standardised residuals indicated that the data contained approximately normally distributed errors, confirmed with the normal P-P plots of standardised residuals, and the scatterplots of standardised residuals showed that the data met the assumptions of homogeneity of variance and linearity.

\section{Results}

Results are presented for each of the research questions.

MANOVA revealed a statistically significant difference in quality of life based on participants' gender, $F(4,460)=3.64$, $p=.006$; Wilk's $\Lambda=.97 ; \eta^{2}=.03$. Gender has a statistically significant effect on the physical $(F(1,463)=6.48 ; p=.01$; $\left.\eta^{2}=.01\right)$ psychological $(F(1,463)=13.47 ; p<.0005$; $\left.\eta^{2}=.03\right)$, and social relations domains $(F(1,463)=5.29$; $\left.p=.02 ; \eta^{2}=.01\right)$, with women presenting poorer quality of life scores than men in these three domains.

No statistically significant differences were found in trauma exposure perceptions based on gender.

A one-way MANOVA revealed a statistically significant difference in quality of life based on participants' age group ( $F$ $(20,1500.06)=4.57, p<.0005$; Wilk's $\left.\Lambda=.82 ; \eta^{2}=.05\right)$. Still, age only had a statistically significant effect on the psychological domain $\left(F(5,455)=8.48 ; p<.0005 ; \eta^{2}=.09\right)$ with younger participants (18-24 years old) presenting poorer psychological quality of life scores than participants in all other age groups.

There were also significant differences in perception of exposure based on the age group of participants $(F(20$, $1383.98)=1.84, p=.013 ;$ Wilk's $\left.\Lambda=.92 ; \eta^{2}=.02\right)$, with a statistically significant effect of age on coping $(F(5,420)=$ $\left.3.80 ; p=.002 ; \eta^{2}=.04\right)$. Again, in this variable the age group $18-24$ presented significantly lower scores in coping compared the age groups 25-34, 35-44, and 55-64.

Statistical analyses revealed a significant difference in quality of life based on marital status $(F(12,1214.67)=$ 
4.35, $p<.0005$; Wilk's $\left.\Lambda=.90 ; \eta^{2}=.04\right)$. Such difference was significant on the psychological $(F(3,462)=5.06, p=.002$; $\left.\eta^{2}=.03\right)$ and social relations domains $(F(3,462)=3.89$, $\left.p=.009 ; \eta^{2}=.03\right)$. Those that reported being single had poorer psychological quality of life compared to participants that were married or lived in civil partnership, while those who were divorced/separated showed poorer social relations scores compared to those who were married/in civil partnership. Likewise, there was a significant difference in perception of exposure to the COVID-19 pandemic based on marital status $(F(12,1122.01)=2.11, p=.014$; Wilk's $\Lambda=.94$; $\eta^{2}=.02$ ), with single participants reporting higher scores in the perception of exposure to losses $(F(3,427)=3.26$, $\left.p=.021 ; \eta^{2}=.02\right)$ compared to participants that were married/in civil partnership.

MANOVA revealed statistically significant differences in quality of life based on the employment status of participants at the end of the COVID-19 confinement period ( $F$ (28, $1638.34)=3.47, p<.0005$; Wilk's $\Lambda=.81 ; \eta^{2}=.05$ ), and this was evident for all four domains: physical $(F(7,457)=3.79$, $\left.p=.001 ; \eta^{2}=.06\right) ;$ psychological $(F(7,457)=9.40$, $\left.p<.0005 ; \eta^{2}=.13\right)$; social relations $(F(7,457)=4.16$, $\left.p<.0005 ; \eta^{2}=.06\right)$; and environment $(F(7,457)=2.96$, $\left.p=.005 ; \eta^{2}=.04\right)$.

Students had significantly lower quality of life compared to employed participants (both self-employed and working for others) and retired participants in the physical dimension. The difference was also significant in social relationships between students and employed participants and between unemployed and employed participants. Furthermore, unemployed participants presented lower scores in psychological and environmental quality of life compared to employed participants. In addition, there were statistically significant differences in the perception of exposure to COVID-19 based on employment status $(F(28,1512.15)=2.33, p<.0005$; Wilk's $\Lambda=.86$; $\left.\eta^{2}=.04\right)$, in particular regarding coping $F(7,422)=5.81$, $p<.0005 ; \eta^{2}=.09$ ), with unemployed and student participants reporting worse perceptions compared to employed participants; and perceptions of adequacy of global measures $F$ (7, $422)=3.36, p=.002 ; \eta^{2}=.05$ ), with differences between employed and unemployed participants (the latter presenting poorer perceptions).

MANOVA also revealed a statistically significant difference in quality of life based on participants' responsibilities towards people who needed care $(F(4,462)=5.30, p<.0005$; Wilk's $\left.\Lambda=.95 ; \eta^{2}=.04\right)$. Having at least one person to care for had a statistically significant effect on the psychological domain $(F(1$, $465)=10.36 ; p=.001 ; \eta^{2}=.02$ ), with those who do not have caring responsibilities showing poorer psychological quality of life scores than those who were caregivers. No statistically significant differences were found in trauma exposure perceptions based on responsibilities towards caregivers.
No statistically significant differences were found in quality of life neither in trauma exposure perceptions based on the age of people under care for those who had responsibilities towards them.

No statistically significant differences were found in quality of life based on exposure to risk of infection at work. Nevertheless, MANOVA showed significant differences in trauma exposure perceptions based on risk of infection at work $\left(F(4,425)=3.26, p=.012\right.$; Wilk's $\left.\Lambda=.97 ; \eta^{2}=.03\right)$, specifically in terms of losses $(F(1,428)=6.60, p=.011$; $\left.\eta^{2}=.02\right)$ and coping $\left(F(1,428)=5.77, p=.017 ; \eta^{2}=.01\right)$, with those that were exposed to risk presenting more exposure to traumatic stressors but better coping.

Concerning the second research question, multiple regression models confirmed that several aspects of participants' perceptions regarding their exposure to COVID-19 significantly predicted all domains of their quality of life with considerable explained variance, especially concerning the psychological and environmental domains of quality of life, as shown in Table 2.

\section{Discussion}

The current study addressed the impact of the state of emergency in Portugal due to the COVID-19 pandemic, considering the influence of sociodemographic characteristics on residents' quality of life and trauma exposure perceptions. Results suggest that, at the end of the first state of emergency in Portugal, women had poorer quality of life scores than men but did not perceive their exposure to the COVID-19 pandemic differently. These results concur with Paulino and colleagues' study (Paulino et al., 2020) and may reflect a Portuguese cultural feature whereby women take on more responsibilities at home and in taking care of other family members (Perista et al., 2016; Stoilova et al., 2020; Torres et al., 2018; Voicu et al., 2009). It is possible that the crisis has emphasized the amount of responsibility assigned to women with an impact on their perceived quality of life. Guadagni et al. (2020) also found that gender differences seem to play a role in the individuals' psychological and behavioral reactions to the isolation period in the COVID-19 pandemic. In their study, females reported significantly higher symptoms of anxiety, depression and distress. These findings about gender differences coping with Covid-19 pandemic seems to enhance gender roles and norms as a sociodemographic characteristic that influences quality of life, namely regarding women's role as caregivers (Guadagni et al., 2020).

Young adults (18-24) were more affected in terms of psychological quality of life than other age groups, and reported less social support during the COVID-19 pandemic. Although daily news reported that this age group was not at high risk concerning COVID-19 symptoms, it is possible that young adults were psychologically more affected during 
Table 2 Multiple linear regression models (stepwise)

\begin{tabular}{|c|c|c|c|c|c|c|c|}
\hline Outcome & Predictors & $\mathrm{R}$ & Adj. $R^{2}$ & $\mathrm{~F}$ & $\mathrm{P}$ & Beta Std. & $\mathrm{p}$ \\
\hline \multirow[t]{3}{*}{ WHOQoL physical Domain } & TEC - Loss & .22 & .21 & 39.29 & .00 & -.35 & .00 \\
\hline & TEC - Support & & & & & .28 & .00 \\
\hline & TEC_C - Global measures & & & & & .19 & .00 \\
\hline \multirow[t]{3}{*}{ WHOQoL psychological domain } & TEC - Support & .22 & .22 & 40.28 & .00 & .35 & .00 \\
\hline & TEC - Loss & & & & & -.31 & .00 \\
\hline & TEC_C - Global measures & & & & & .17 & .00 \\
\hline \multirow[t]{3}{*}{ WHOQoL social relations } & TEC - Support & .15 & .14 & 24.47 & .00 & .37 & .00 \\
\hline & TEC - Loss & & & & & -.14 & .00 \\
\hline & TEC - Local measures & & & & & .09 & .05 \\
\hline \multirow[t]{4}{*}{ WHOQoL environment } & TEC_C - Global measures & .27 & .26 & 38.00 & .00 & .26 & .00 \\
\hline & TEC - Loss & & & & & -.31 & .00 \\
\hline & TEC - Support & & & & & .25 & .00 \\
\hline & TEC_C - Local measures & & & & & .13 & .01 \\
\hline
\end{tabular}

confinement due to the loss of social relations, and greater isolation, which disrupted their routines and active way of life (Hou et al., 2020). Younger adults with ages comprising 18 24 years tend to be involved in non-cohabitation intimate relationships. Therefore, it is possible that during the confinement period they also had less emotional and physical contact with intimate partners and other significant social relationships. Social activities and hobbies were also largely suspended as gymnasiums, coffees, restaurants, theatres, cinemas, and nightclubs were closed. Additionally, university classes only occurred online, and face-to-face contact was highly reduced.

Indeed, the importance of meaning-making (Milman et al., 2020a; Milman et al., 2020b; Norris \& Wind, 2010; Schulenberg, 2016) appears relevant both in the case of women and young adults, as the meaning attributed to this experience in terms of burdens and losses may have contributed for the decrease in their quality of life.

Single participants showed lower psychological quality of life than those that reported being married or in a civil partnership. Likewise, divorced participants showed poorer social relations than those that reported being married or in a civil partnership. This suggests a protective effect of having a spouse/partner in these aspects of quality of life. In the same sense, those who were single reported worse perceptions in terms of traumatic stressors relating to the COVID-19 pandemic than those who were married/in civil partnership. It is possible that a large number of single participants lived alone at home, where they spent the confinement period during the emergency state, which significantly impaired the opportunity for social and affective contacts with other individuals. This result is in line with other studies on the impact of the pandemic, that mention the role of isolation and lack of support as sources of increased vulnerability (Pedrosa et al., 2020).
Results on students' quality of life, considered along with results from younger adults, and single participants, add to the possibility that young adults may have been the most affected psychosocially (compared to other age groups), having lost more activities and having been more isolated than others, which would explain their poorer quality of life perceptions, and lower perception of support even in comparison with retired/older participants.

Likewise, the fact that those who had people to care for, showed significantly better quality of life in the psychological domain suggest that, despite the weight of responsibility, having others to look after, protected carers from isolation and poorer psychological outcomes. The sense of purpose or responsibility for taking care of others may have acted as a protective factor during this particular confinement period. It is also possible that having to keep a daily routine for others provided a sense of predictability and of coherence that protected this group of individuals (Hou et al., 2020). Moreover, an appropriate sense of coherence and predictability may enhance individuals' narratives about stressful events, such as those surrounding the COVID-19 pandemic, and promote a healthier reconstruction of meaning regarding burden and loss (Castiglioni \& Gaj, 2020). Although this study represents a specific time period, when the pandemic was being experienced by Portuguese residents as a relatively short experience (from March to May), the burden and losses associated with the COVID-19 pandemic are still ongoing for a yet indefinite period. This means that, for over a year, individuals have been and continue to be deprived from a sense of predictability and of coherence, which is associated with an experience of psychological fatigue (Morgul et al., 2020). In line with this idea, the processes of meaning reconstructing, with emphasis on metaphors, narratives and a sense of coherence (Castiglioni \& Gaj, 2020), as well as a daily routine appear vital for health promotion in the short and long term. 
As expected, unemployment appears to be a risk factor for poorer quality of life in psychological and environmental domains, as well as for poorer coping and adequacy of global measures to tackle the pandemic.

Having been exposed to risk of COVID-19 infection at work was related to worse perceptions of exposure to traumatic stressors but to better coping, not having a significant impact on participants' quality of life. Again, the role of social support may have moderated the stressors of those potentially exposed to infection at work (Gil-Rivas \& Kilmer, 2016; Norris et al., 2008). This result may evidence the success of some health measures that took place in Portugal and the support and sense of security felt by people exposed to the virus. Indeed, at this time, the Portuguese authorities implemented measures to respond to the challenges of the pandemic in several domains: economy, education, and heathcare. Regarding the latter, the National Health Service (SNS) created a free telephone service with nurses and psychologists, to address questions, symptoms, and worries of the general population. Locally, some health administration authorities have also organised integrated responses to stop COVID-19 transmission chains, involving doctors, nurses, dentists, teachers, psychologists and military personnel.

These results on individuals exposed to risk of COVID-19 infection at work are unexpected, to some degree. It is possible that they may relate to the resources people activate to face the emergency and the meaning given to life during a traumatic event (Negri et al., 2020). Milman at al. (Milman et al., 2020a) suggest that meaning- making of the COVID-19 pandemic may not be entirely an intrapsychic process, but also reliant on social and cultural meaning values, such as engaging in normative social prescriptions and approved guidelines to mitigate COVID-19 transmission.

Results also allow to conclude that the degree of exposure to COVID-19 (perceived at individual and community levels) contributes to explain quality of life outcomes. Indeed, results suggest that the perception of bigger losses (more exposure to traumatic stressors) have a negative impact on all domains of quality of life. Likewise, better perceived support from family, friends, and communities was found to contribute to better quality of life results in the physical, psychological, and environmental domains. In line with these results, a study with pregnant women who delivered during the COVID-19 pandemic showed that these women reported lower risk of developing post-partum depression compared to women who delivered before the pandemic crisis (Pariente et al., 2020), highlighting the role of family support and cohesion, and emotional bounds with intimate partners as protective factors during crisis events. Intriguingly, this perception of support was not found as a significant predictor for better quality of life in terms of social relations. It is possible that perceived support from family, friends, and communities was mainly related to health care, financial and logistic support, and not so much associated with emotional and affective experiences. This would be consistent with conclusions from a study on the impact of the COVID-19 pandemic (Zysberg \& Zisberg, 2020) suggesting that psychological-emotional social support may play a different role in individuals' well-being compared to instrumental support. In addition, the social relations domain of the WHOQoL focuses on personal relations, sexual intercourse and support from friends. Recent studies showed that the quality of sexual life during COVID-19 pandemic was significantly impaired (e.g., Schiavi et al., 2020; Yuksel \& Ozgor, 2020), with a decrease in sexual activity frequency and sexual function, and an increase in sexual distress (Schiavi et al., 2020). The lack of quality of sexual life along with intimate partners living apart or being single, may help explain the fact that perceived support from family, friends, and communities was not found as a significant predictor for better quality of life in terms of social relations.

Regarding the appraisal of measures to tackle the COVID19 pandemic, the perception of efficacy and adequacy of more global measures (government, healthcare, education, etc.) had a significant impact on quality of life in terms of physical, psychological and environmental domains, whereas the perception of adequacy of measures implemented locally had an impact on social relations and on the environmental domain of quality of life.

Regardless of the relevance of current findings, the present study has some limitations and results should be generalized with caution. This study was conducted with a sample collected online. Although during this confinement period no face-toface sample collection was allowed, only individuals with internet access and who felt comfortable with web-surveys were able to participate. The sample was mainly comprised of women, which may impair the results of the multivariate and regression analysis. This overrepresentation of female participants was also found in a recent Portuguese study (Paulino et al., 2020). The magnitude of the values observed was small, possibly due to other unaccounted factors that contribute to quality of life and exposure to traumatic experiences. Nevertheless, results enhance the existence of protective individual and community characteristics that can be promoted in a crisis situation.

\section{Conclusions}

Overall, the present study suggests that, during the generalised confinement period, those that had work or caring responsibilities to focus on and those who lived with others (spouses, caregivers) were more protected from the psychosocial challenges of the COVID-19 pandemic, not only in terms of quality of life, but also in terms of their exposure to this potentially traumatic experience.

Perceptions regarding government measures to deal with the crisis were also found to have a positive impact in the quality of 
life, as suggested by results from participants who were exposed to risk of infection at work and by the significant impact that perceptions regarding governmental measures on quality of life (with global measures impacting physical, psychological and environmental domains and local measures having an impact on social and environmental domains. The relevance of the macro-context (Hoffman \& Kruczek, 2011; Morgado, 2020) and the feeling of belonging to a country that appears to care about citizens seem to be protective factors when dealing with a crisis, such as the COVID-19 pandemic.

This study aimed to identify the implications of the generalised confinement period for different sociodemographic groups. Further studies should seek to target populations, such as health professionals, students, and the unemployed, to understand how their living/occupational conditions can be further impacted by the COVID-19 pandemic. Future investigations should also consider other variables to understand how people cope with this crisis, using measures to assess psychopathological symptomatology, for example, of depression, anxiety, and PTSD (Brooks et al., 2020; Duan \& Zhu, 2020; Rajukmar, 2020). The risk perception related to the personal wellbeing is also a concern during this pandemic. As subjective factors affect the risk perception and the perceived susceptibility, it may be interesting to study psychological wellbeing of people during Covid crisis and its relations with preventive and control policies and well as health education measures (Commodari et al., 2020; Ding et al., 2020).

Considering the unique experience being lived during the COVID-19 pandemic, a longitudinal study could contribute to gain knowledge regarding the way people keep coping with the constant challenges that are arising, as well as with the evolution of knowledge and clinical responses to the virus.

Finally, this study suggests that mental health services should promote responses to specific populations that are more vulnerable to crises such as the COVID-19 pandemic, namely individuals living alone, young adults, and women. It also advocates that in order to foster the adaptive functioning of individuals and communities, European, national and local measures must be well conveyed, ensuring that the population is able to access and understand information. This may allow individuals to develop more positive perceptions on their exposure to the pandemic, as well to have better opportunities for meaning making that will contribute towards quality of life during times of change and uncertainty. This may help individuals and communities to restore their belief in personal core views about the world, their sense of resilience and predictability and, consequently, enhance health globally.

\section{Summary}

The current study explored the impact of the state of emergency due to COVID-19 pandemic in the Portuguese context on individuals' perceptions regarding their exposure to the pandemic and their quality of life, taking into consideration sociodemographic characteristics such as gender, age, marital status, employment status, and caring responsibilities.

Women reported poorer quality of life at the end of the confinement period than men. Young and single participants had poorer quality of life and reported worse perceptions of the COVID-19 pandemic as a traumatic experience. Being unemployed was also associated with poorer quality of life and worse perceptions of the exposure to the COVID-19 pandemic. The same applies to individuals who did not have people to care for.

Perceptions on governmental measures to handle the crisis and on support from family, friends and community had a positive impact on quality of life, whereas perceptions of exposure to traumatic stressors had a negative impact on quality of life.

European and country-wide measures to tackle mental health challenges in the near future should consider sociodemographic characteristics of the population in order to promote better levels of quality of life and diminish the impact of exposure to traumatic events. Efforts should be particularly targeted towards risk groups such as women, younger and single adults, unemployed, and individuals who did not have others to care for.

Data Availability The datasets generated during and/or analysed during the current study are not.

publicly available due to further data analysis in the scope of the ongoing project. Datasets may be made available in the future from the corresponding author on reasonable request.

\section{Declarations}

Ethical Statement All procedures performed were in accordance with the ethical standards of the institutional ethics committee and with the 1964 Helsinki declaration and its later amendments. The current study is part of a research project approved by Ordem dos Psicólogos Portugueses (Portuguese Psychologists Board) and by the institutional ethics committee of the authors' institution of affiliation.

Informed Consent Informed consent was obtained from all individual participants included in the study.

Conflict of Interest The authors declare no conflict of interest.

\section{References}

Allen, P., \& Bennett, K. (2008). SPSS for the health and behavioural sciences. Thomson.

Asmundson, G., \& Taylor, S. (2020). How health anxiety influences responses to viral outbreaks like COVID-19: What all decisionmakers, health authorities, and health care professionals need to know. Journal of Anxiety Disorders, 71, 102211. https://doi.org/ 10.1016/j.janxdis.2020.102211. 
Benight, C. C., Cieslak, R., \& Waldrep, E. (2010). Social and cognitive frameworks for understanding the mental health consequences of disasters. In Y. Neria, S. Galea, \& F. H. Norris (Eds.), Mental health and disasters (pp. 161-174). Cambridge University Press. https:// doi.org/10.1017/CBO9780511730030.010.

Biviá-Roig, G., la Rosa, V. L., Gómez-Tébar, M., Serrano-Raya, L., Amer-Cuenca, J. J., Caruso, S., Commodari, E., Barrasa-Shaw, A., \& Lisón, J. F. (2020). Analysis of the impact of the confinement resulting from COVID-19 on the lifestyle and psychological wellbeing of Spanish pregnant women: An internet-based cross-sectional survey. International Journal of Environmental Research and Public Health, 17, 5933. https://doi.org/10.3390/ijerph17165933.

Bonanno, G.A., \& Gupta, S. (2010). Resilience after disaster. In Y. Neria, S. Galea, \& F.H. Norris (Eds.), Mental health and disasters (pp. 145160). Cambridge: Cambridge University Press. https://doi.org/10. 1017/CBO9780511730030.009.

Brooks, S. K., Webster, R. K., Smith, L. E., Woodland, L., Wessely, S., Greenberg, N., \& Rubin, G. J. (2020). The psychological impact of quarantine and how to reduce it: Rapid review of the evidence. The Lancet, 395, 912-920. https://doi.org/10.1016/S0140-6736(20)30460-8.

Castiglioni, M., \& Gaj, N. (2020). Fostering the reconstruction of meaning among the general population during the COVID-19 pandemic. Frontiers in Psychology, 11, 567419. https://doi.org/10.3389/fpsyg. 2020.567419.

Coelho, C. M., Suttiwan, P., Arato, N., \& Zsido, A. N. (2020). On the nature of fear and anxiety triggered by COVID-19. Frontiers in Psychology, 11, 581314. https://doi.org/10.3389/fpsyg.2020. 581314.

Commodari, E., La Rosa, V. L., \& Coniglio, M. A. (2020). Health risk perceptions in the era of the new coronavirus: Are the Italian people ready for a novel virus? A cross-sectional study on perceived personal and comparative susceptibility for infectious diseases. Public Health, 187, 8-14. https://doi.org/10.1016/j.puhe.2020.07.036.

Cox, R. S., \& Perry, K. E. (2011). Like a fish out of water: Reconsidering disaster recovery and the role of place and social capital in community disaster resilience. American Journal of Community Psychology, 48, 395-411. https://doi.org/10.1007/s10464-011-9427-0.

Ding, Y., Xu, J., Huang, S., Li, P., Lu, C., \& Xie, S. (2020). Risk perception and depression in public health crises: Evidence from the COVID-19 crisis in China. International Journal of Environmental Research and Public Health, 17(16), 5728. https://doi.org/10.3390/ ijerph17165728.

Duan, L., \& Zhu, G. (2020). Psychological interventions for people affected by the COVID-19 epidemic. Lancet Psychiatry, 7, 300-302. https://doi.org/10.1016/S2215-0366(20)30073-0.

European Centre for Disease Prevention and Control (2020). Country response measures to COVID-19 [data file]. Retrieved from: https://www.ecdc.europa.eu/en/publications-data/download-dataresponse-measures-covid-19

Eysenbach, G. (2004). Improving the quality of web surveys: The checklist for reporting results of internet e-surveys (CHERRIES). Journal of Medical Internet Research, 6(3), e34. https://doi.org/10.2196/ jmir.6.3.e34.

Field, A. (2018). Discovering statistics using IBM SPSS statistics (5th ed.). SAGE.

Gil-Rivas, V., \& Kilmer, R. (2016). Building community capacity and fostering disaster resilience. Journal of Clinical Psychology, 72(12), $1318-1332$.

Guadagni, V., Umilta, A., \& Iaria, G. (2020). Sleep quality, empathy, and mood during the isolation period of the COVID-19 pandemic in the Canadian population: Females and women suffered the most. Frontiers in Global Women's Health, 1, 585938. https://doi.org/ 10.3389/fgwh.2020.585938.

Hoffman, M., \& Kruczek, T. (2011). A bioecological model of mass trauma: Individual, community, and societal effects. The
Counseling Psychologist, 39(8), 1087-1127. https://doi.org/10. 1177/0011000010397932.

Hou, W. K., Lai, F. T. T., Ben-Ezra, M., \& Goodwin, R. (2020). Regularizing daily routines for mental health during and after the COVID-19 pandemic. Journal of Global Health, 10(2), 020315. https://doi.org/10.7189/jogh.10.020315.

Milman, E., Lee, S. A., \& Neimeyer, R. A. (2020a). Social isolation and the mitigation of coronavirus anxiety: The mediating role of meaning. Death Studies. https://doi.org/10.1080/07481187.2020. 1775362.

Milman, E., Lee, S. A., Neimeyer, R. A., Mathis, A. A., \& Jobe, M. C. (2020b). Modeling pandemic depression and anxiety: The mediational role of core beliefs and meaning making. Journal of Affective Disorders Reports, 2, 100023. https://doi.org/10.1016/j.jadr.2020. 100023.

Morgado, A. M. (2020). Disasters, individuals, and communities: Can positive psychology contribute to community development after disaster? Community Development, 51(1), 3-16. https://doi.org/10. 1080/15575330.2020.1714685.

Morgado, A. M., Cruz, J., \& Peixoto, M. (2021). Trauma Exposure Checklist: Psychometric properties and its applicability to the COVID-19 pandemic. Journal of Loss and Trauma. https://doi. org $/ 10.1080 / 15325024.2021 .1892408$.

Morgul, E., Bener, A., Atak, M., Akyel, S., Aktaș, S., Bhugra, D., Ventriglio, A., \& Jordan, T. R. (2020). COVID-19 pandemic and psychological fatigue in Turkey. The International Journal of Social Psychiatry. https://doi.org/10.1177/0020764020941889.

Negri, A., Andreoli, G., Barazzetti, A., Zamin, C., \& Christian, C. (2020). Linguistic markers of the emotion elaboration surrounding the confinement period in the Italian epicenter of COVID-19 outbreak. Frontiers in Psychology, 11, 568281. https://doi.org/10.3389/ fpsyg.2020.568281.

Neimeyer, R. A., Burke, L., Mackay, M., \& Stringer, J. (2010). Grief therapy and the reconstruction of meaning: From principles to practice. Journal of Contemporary Psychotherapy, 40, 73-83. https:// doi.org/10.1007/s10879-009-9135-3.

Norris, F., Stevens, S., Pfefferbaum, B., Wyche, K., \& Pfefferbaum, R. (2008). Community resilience as a metaphor, theory, set of capacities, and strategy for disaster readiness. American Journal of Community Psychology, 41, 127-150.

Norris, F., \& Wind, L. (2010). The experience of disaster: Trauma, loss, adversities, and community effects. In Y. Neria, S. Galea, \& F. H. Norris (Eds.), Mental health and disasters (pp. 29-44). Cambridge University Press.

Pariente, G., Wissotzky Broder, O., Sheiner, E., Lanxner Battat, T., Mazor, E., Yaniv Salem, S., Kosef, T., \& Wainstock, T. (2020). Risk for probable post-partum depression among women during the COVID-19 pandemic. Archives of Women's Mental Health, 23, 767-773. https://doi.org/10.1007/s00737-020-01075-3.

Paulino, M., Dumas-Diniz, R., Brissos, S., Brites, R., Alho, L., Simões, M. R., \& Silva, C. F. (2020). COVID-19 in Portugal: Exploring the immediate psychological impact on the general population. Psychology, Health \& Medicine, 26, 44-55. https://doi.org/10. 1080/13548506.2020.1808236.

Pedrosa, A. L., Bitencourt, L., Fróes, A. C. F., Cazumbá, M. L. B., Campos, R. G. B., Brito, S. B. C. S., \& Silva, A. C. S. (2020). Emotional, Behavioral, and Psychological Impact of the COVID19 Pandemic. Frontiers in Psychology, 11, 566212. https://doi.org/ 10.3389/fpsyg.2020.566212.

Pérez-Sales, P., Cervellón, P., Vázquez, C., Vidales, D., \& Gaborit, M. (2005). Post-traumatic factors and resilience: The role of shelter management and survivors' attitudes after the earthquakes in El Salvador (2001). Journal of Community \& Applied Social Psychology, 15, 368-382. 
Perista, H., Cardoso, A., Brázia, A., Abrantes, M., Perista, P., \& Quintal, E. (2016). The use of time by men and women in Portugal. CESIS and CITE.

Rajukmar, R. P. (2020). COVID-19 and mental health: A review of the existing literature. Asian Journal of Psychiatry, 52, 102066. https:// doi.org/10.1016/j.ajp.2020.102066.

Raphael, B., \& Maguire, P. (2010). Disaster mental health research: Past, present, and future. In Y. Neria, S. Galea, \& F. H. Norris (Eds.), Mental health and disasters (pp. 7-28). Cambridge University Press.

Schiavi, M. C., Spina, V., Zullo, M. A., Colagiovanni, V., Luffarelli, P., Rago, R., \& Palazzetti, P. (2020). Love in the time of COVID-19: Sexual function and quality of life analysis during the social distancing measures in a group of Italian reproductive-age women. Journal of Sexual Medicine, 17, 1407-1413. https://doi.org/10.1016/j.jsxm. 2020.06.006.

Schulenberg, S. (2016). Disaster mental health and positive psychology considering the context of natural and technological disasters: An introduction to the special issue. Journal of Clinical Psychology, 72(12), 1223-1233. https://doi.org/10.1002/jclp.22409.

Stoilova, R., Ilieva-Trichokova, P., \& Bieri, F. (2020). Work-life balance in Europe: Institutional contexts and individual factors. International Journal of Sociology and Social Policy, 40(3/4), 366-381. https://doi.org/10.1108/IJSSP-08-2019-0152.

Torres, A., Pinto, P. C., Costa, D., Coelho, B., Maciel, D., Reigadinha, T., \& Theodoro, E. (2018). Igualdade de género ao longo da vida: Portugal no contexto europeu. Fundação Francisco Manuel dos Santos.

Vaz Serra, A., Canavarro, M. C., Simões, M., Pereira, M., Gameiro, S., Quartilho, M., \& Paredes, T. (2006). Estudos psicométricos do instrumento de Avaliação da Qualidade de Vida da Organização Mundial de Saúde (WHOQOL-Bref) para Português de Portugal. Psiquiatria Clínica, 27(1), 41-49.

Venuleo, C., Marinaci, T., Gennaro, A., \& Palmieri, A. (2020). The meaning of living in the time of COVID-19. A large sample narrative inquiry. Frontiers in Psychology, 11, 577077. https://doi.org/ 10.3389/fpsyg.2020.577077.

Verdery, A. M., \& Smith-Greenaway, E. (2020). COVID-19 and family bereavement in the United States. Applied Demography - Special Issue on COVID-19, 32(1), 1-2.

Voicu, M., Voicu, B., \& Strapcova, K. (2009). Housework and gender inequality in European countries. European Sociological Review, 25(3), 365-377. https://doi.org/10.1093/esr/jcn054.

Yuksel, B., \& Ozgor, F. (2020). Effect of the COVID-19 pandemic on female sexual behavior. International Journal of Gynecology \& Obstetrics, 150, 98-102. https://doi.org/10.1002/ijgo.13193.

Zhang, J., Wu, W., Zhao, X., \& Zhang, W. (2020). Recommended psychological crisis intervention response to the 2019 novel coronavirus pneumonia outbreak in China: A model of West China Hospital. Precision Clinical Medicine, 3, 3-8. https://doi.org/10.1093/ pcmedi/pbaa006.

Zysberg, L., \& Zisberg, A. (2020). Days of worry: Emotional intelligence and social support mediate worry in the COVID-19 pandemic. Journal of Health Psychology. https://doi.org/10.1177/ 1359105320949935.

Publisher's Note Springer Nature remains neutral with regard to jurisdictional claims in published maps and institutional affiliations. 